\title{
Padronização e teste da plataforma de força em padrão ortostático em cães
}

\author{
[Test and standardize of force platelet in ortostatic pattern in dogs] \\ A.L.T. Barbosa ${ }^{1}$, J.E.W. Schossler ${ }^{2}$, C.M. Bolli ${ }^{2}$, L.F.C. Lemos ${ }^{3}$, C. Medeiros ${ }^{3}$ \\ ${ }^{1}$ Aluna de pós-graduação - Universidade Federal de Santa Maria - Santa Maria, RS \\ ${ }^{2}$ Universidade Federal de Santa Maria - Santa Maria, RS \\ ${ }^{3}$ Alunos de graduação - Universidade Federal de Santa Maria - Santa Maria, RS
}

\begin{abstract}
RESUMO
O objetivo deste trabalho foi padronizar e testar o uso da plataforma de força em padrão ortostático em cães, na avaliação do apoio e distribuição do peso nos membros, permitindo visualizar uma possível transferência em pacientes ortopédicos com afecções articulares. Foram realizadas avaliações clínica - graus de claudicação e exame radiográfico - e biomecânica na plataforma de força, por coleta estática de 16 pacientes cirúrgicos ortopédicos com afecções coxofemoral da rotina hospitalar (grupo tratado), e de seis cães saudáveis, sem histórico de problemas ortopédicos (grupo-controle). Com base no grupo-controle, foi possível delinear uma metodologia adequada para cães em coleta estática e testá-la no grupo tratado. A plataforma em padrão ortostático mostrou ser um parâmetro objetivo, eficiente e mais sensível que os demais, sendo seu uso adequado na avaliação de pacientes ortopédicos.
\end{abstract}

Palavras-chave: cão, placa de força estática, transferência de peso

\begin{abstract}
The purpose of this work was to standardize and test the use of the force platform in ortostatic pattern in dogs, to approach the support assessment and weight distribution in the limbs, allowing the visualization of the transfer possibility in surgical orthopedics patients with hip diseases. Clinical evaluation - lameness degrees and radiografic exam - and biomechanical evaluation were accomplished through the force plate for static collection of 16 surgical orthopedics patients with hip diseases in hospital routine (control-group) and 6 healthy dogs without a report of orthopedics problems (treatment-group). The control-group for outline allowed an appropriate methodology at static collection in dogs. The platform in ortostátic pattern was efficient and a more sensitive and objective parameter than the others, being of appropriate use in the evaluation of an orthopedic patient.
\end{abstract}

Keywords: dog, force platform static, weight transfer

\section{INTRODUÇÃO}

A plataforma de força tem sido usada como avaliação objetiva e não invasiva da marcha normal e anormal em humanos e animais (Dogan et al., 1991). A ortopedia humana têm usado a plataforma na mensuração de forças externas antes e depois do tratamento dos pacientes há duas décadas (Anderson e Mann, 1994).

Vários estudos têm usado a plataforma de força em animais para avaliação da marcha em especialidades da medicina veterinária como

Recebido em 23 de abril de 2010

Aceito em 12 de abril de 2011

E.mail: laeticiatrindade@yahoo.com.br neurologia e ortopedia. Wheeler e Raiser (1992) realizaram um estudo cinemático da deambulação de cães submetidos à artroplastia capsular da articulação coxofemoral para se obter a avaliação quantitativa da técnica cirúrgica empregada. O exame biomecânico, comparado à avaliação clínica demonstrou ser um método objetivo (quantitativo) para análise de alterações na locomoção. Jevens et al. (1996) usaram a plataforma na comparação de duas técnicas cirúrgicas no tratamento de ruptura do ligamento cruzado cranial em cães, e Bertram et al. (2000) compararam a marcha durante o trote de duas raças: Labrador Retrivers e Greyhounds. 
Conzemius et al. (2003) estudaram a função do membro antes e depois da artroplastia total do cotovelo acometido por osteoartrite severa e natural, enquanto Van Klaveren et al. (2005) usaram a análise na plataforma de força, antes e depois da descompressão, como tratamento da estenose lombossacra degenerativa em cães. Evans et al. (2005) avaliaram a acurácia e otimização da plataforma de força na análise da marcha em Labradores com afecções no ligamento cruzado cranial, avaliados pela claudicação em marcha. Observaram, após a cirurgia, que $75 \%$ dos animais que não apresentavam claudicação evidenciaram déficit na análise da plataforma e concluíram que a plataforma é um método acurado de avaliação de claudicação dessa raça com ruptura de ligamento cruzado cranial e que é mais sensível que a observação visual.

Suwankong et al. (2007) avaliaram, em longo prazo, a cirurgia de descompressão em cães com estenose lombossacra degenerativa por intermédio da análise da plataforma de força, enquanto Bockstahler et al. (2007) estudaram a detecção de mudanças cinemáticas, com ou sem detecção radiográfica de displasia coxofemoral. Concluíram que a plataforma é um método, em longo prazo, mais sensível que o exame radiográfico na detecção de alterações displásicas na articulação coxofemoral subclínicas ainda não detectadas pela radiografia.

A medicina humana, além de avaliar a marcha na plataforma de força, tem realizado estudos estabilométricos para julgar a postura e o equilíbrio, utilizando a plataforma em padrão ortostático. Oliveira (1993) descreveu que a estabilometria avalia o equilíbrio postural por meio da quantificação da oscilação postural na posição ortostática na plataforma de força. Geralmente os testes são aplicados sobre diferentes protocolos para a base de suporte pés juntos, afastados, apoio em um só pé, etc. superfície - dura ou espuma - e a visão - olhos abertos ou fechados. A estabilidade é alcançada ao gerar momentos de força sobre as articulações do corpo para neutralizar o efeito da gravidade ou qualquer outra perturbação, em um processo contínuo e dinâmico, durante a permanência em determinada postura. A oscilação do corpo durante a postura ereta é usualmente investigada utilizando-se uma plataforma de força (Duarte, 2000).
Aferências de receptores plantares e proprioceptivos fornecem ao sistema nervoso informações necessárias ao controle postural. Alterações desses mecanismos neuromusculares provocadas por traumas podem causar dano articular e alterações posturais permanentes (Teodori et al., 2005).

Teodori et al. (2005) avaliaram as modificações na distribuição da pressão plantar e localização do centro de força em sujeito com história de entorse de tornozelo direito, utilizando uma plataforma de pressão Tekscan-Matscan, em apoio bipodal livre e com os olhos abertos. Após constatação de assimetria na distribuição da pressão plantar, realizaram uma sessão de reeducação postural global (RPG), seguida de avaliações em plataforma de pressão, imediatamente após a intervenção desta e após sete, 14 e 30 dias. Os resultados mostraram recuperação evidente da simetria, que se manteve por sete dias. Após esse período, houve tendência à recuperação gradativa da assimetria, não alcançando, no entanto, os valores iniciais após 30 dias.

Trípoli et al. (2008) afirmaram que o reconhecimento da assimetria na distribuição do peso corpóreo em ortostatismo, de indivíduos hemiparéticos e a capacidade de redistribuição de peso constituem um importante aspecto para reabilitação. Por isto, analisaram a transferência de peso durante tarefas orientadas e após feedbacks em hemiparéticos para avaliar a redistribuição do peso nos diversos posicionamentos.

Ao contrário da medicina humana, que tem usado a plataforma também em estudos estabilométricos para avaliar a postura e o equilíbrio (Bastos et al., 2005; Ferreira, 2005; Loth, 2007), na veterinária o uso da plataforma restringe-se à coleta de dados em marcha para análise locomotora. Dessa forma, não foram encontrados na literatura consultada trabalhos que fizeram uso da plataforma em coleta estática, ou seja, em um padrão ortostático para o enfoque na avaliação do apoio e distribuição do peso nos membros, o que permitiria a visualização de possível transferência.

Os objetivos deste trabalho foram: padronizar a metodologia do uso da plataforma de força em coleta estática, ou seja, em padrão ortostático, 
para o enfoque na avaliação do apoio e distribuição do peso nos membros, a qual permita a visualização de possível transferência, e testar o seu uso neste padrão como parâmetro objetivo de avaliação em pacientes ortopédicos com afecções articulares.

\section{MATERIAL E MÉTODOS}

A metodologia foi dividida em duas etapas: padronização e teste da plataforma de força. Foi formado um grupo-controle, constituído de seis animais adultos, sem raça definida, saudáveis, de porte semelhante (aprox. 10kg), sem histórico de problemas ortopédicos ou demais patologias que pudessem comprometer o estudo. Esse grupo teve o objetivo de fornecer os parâmetros normais, encontrados em animais saudáveis, na avaliação da plataforma de força para permitir uma padronização.

Para o teste da plataforma em padrão ortostático, foi formado um grupo tratado, constituído de 16 pacientes ortopédicos da rotina hospitalar, pesando de 8 a 15kg, anteriormente submetidos à cirurgia da articulação coxofemoral colocefalectomia - e à inserção de pino transacetabular após doenças em que esses métodos poderiam ser curativos. Esse grupo foi subdividido em três subgrupos de animais, em que a cirurgia ocorreu até dois meses antes, de dois meses a dois anos ou há mais de dois anos.

Os grupos controle e tratado foram submetidos à avaliação clínica, à avaliação do apoio na plataforma de força em padrão ortostático e ao exame radiográfico. No grupo tratado, a avaliação clínica constituiu-se de anamnese e dos graus de claudicação. O enfoque da anamnese foi: data da cirurgia, membro afetado, cirurgia específica e recuperação do paciente, segundo o proprietário. Os graus de claudicação foram avaliados segundo Braden e Brinker (1973), modificados de acordo com a Tab. 1. No grupocontrole, a avaliação clínica foi constituída de anamnese e exame físico, somente para certificar a normalidade clínica e deambulatória dos animais.

Tabela 1. Características clínicas dos cinco graus de claudicação utilizados para verificar a recuperação do uso funcional do membro dos cães avaliados após cirurgia na articulação coxofemoral

\begin{tabular}{cl}
\hline Grau & \multicolumn{1}{c}{ Característica } \\
\hline I & Não há uso nem apoio do membro. \\
II & $\begin{array}{l}\text { Não há suporte de peso em estação.Uso claudicante ao caminhar, elevando-o ao correr. } \\
\text { Não há suporte de peso em estação. Uso normal do membro ao caminhar, às vezes } \\
\text { III }\end{array}$ \\
claudicando ao correr. & $\begin{array}{l}\text { Uso e apoio normal em estação e ao caminhar, às vezes claudicando ao correr. } \\
\text { Vormal. Uso funcional do membro em estação, caminhando e correndo, com suporte total de } \\
\text { peso. }\end{array}$ \\
\hline
\end{tabular}

Para avaliação do apoio, coletaram-se os dados de cada animal sobre uma plataforma de força na posição ortostática (em estação), em nove posicionamentos diferentes: quatro patas (Fig. 1A); lado direito (D, Fig. 1B); lado esquerdo (E, Fig. 1C); dianteiras (F, Fig. 1D); traseiras (T, Fig. 1E); dianteira direita (DD, Fig. 1F); dianteira esquerda (DE, Fig. 1G); traseira direita (TD, Fig. 1H); e traseira esquerda (TE, Fig. 1I). Em cada posicionamento, o animal deveria estar completamente parado durante cinco segundos, para a coleta dos dados. Foram realizadas três repetições em cada posicionamento, com intervalo de 30 segundos entre coletas, em que o animal descia da plataforma e esta era zerada.

Arq. Bras. Med. Vet. Zootec., v.63, n.3, p.559-566, 2011
Os dados foram coletados por meio de uma plataforma de força OR6-6 AMTI (Advanced Mechanical Technologies, Incorporation, USA) (Fig. 1J), nivelada ao piso, medindo 464x508x82,5mm, equipada com placa analógica digital ADI 32, amplificador Mas-6 e software específico, localizada no Laboratório de Pesquisa e Ensino do Movimento Humano - Núcleo de Biomecânica do Centro de Educação Física e Desportos da Universidade Federal de Santa Maria, e operada por um profissional da área.

A plataforma realizava a quantificação da magnitude das forças de reação do solo (eixos X, $\mathrm{Y}$ e Z) por meio de transdutores do tipo strain gages fixados em células de carga, localizados nos quatro cantos da plataforma, com frequência 
de aquisição de dados de $100 \mathrm{~Hz}$. Após coletados, os dados eram armazenados e analisados por um programa específico, Bioanalysis ${ }^{\circledR}$, no padrão Balance, e transferidos para o programa Microsoft Excel ${ }^{\circledR}$, no qual o resultado final do tratamento dos dados designava a porcentagem média de peso que cada animal apresentou em cada um dos nove posicionamentos.

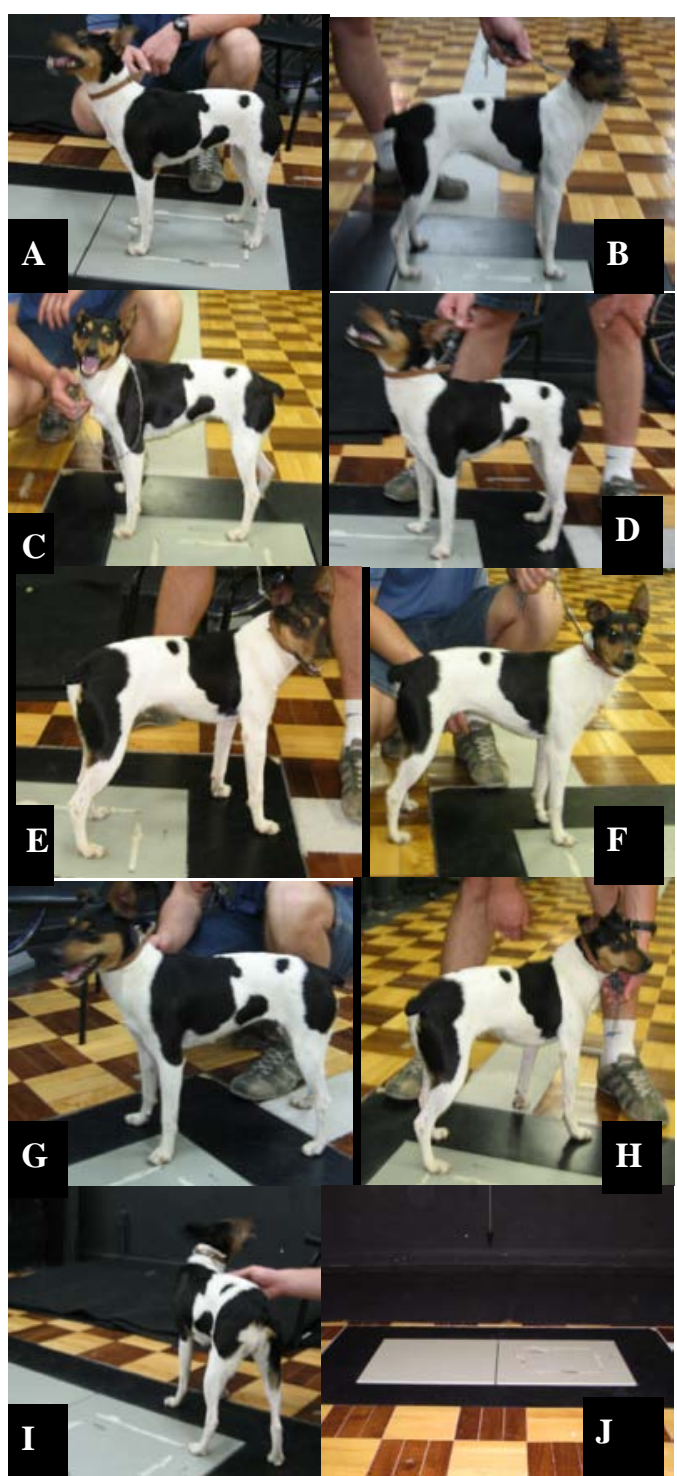

Figura 1. Coleta de dados na plataforma de força nos nove posicionamentos diferentes, com o animal sendo contido por seu proprietário para ilustração do procedimento. A - quatro patas; B - lado direito; $\mathrm{C}$ - lado esquerdo; D - dianteiras; E - traseiras; F - dianteira direita; G - dianteira esquerda; H - traseira direita; I - traseira esquerda; J - vista lateral da plataforma de força.
O primeiro posicionamento, quatro patas, foi utilizado para a normalização dos dados em função do peso corporal. Durante a coleta, os animais foram posicionados sempre por duas pessoas, que participaram de todas as coletas, para garantir o posicionamento adequado dos animais, e mantidos estáticos e alinhados, sempre com o corpo em linha reta e a cabeça voltada para frente. Essa avaliação foi igual em todos os animais.

O exame radiográfico foi realizado em dois posicionamentos, ambos na posição ventrodorsal: o primeiro com os membros em flexão e rotação lateral; o segundo, com os membros estendidos, tracionados e em rotação medial.

Foi realizada estatística descritiva, para obtenção da média e do desvio-padrão dos dados da plataforma de força do grupo-controle, usando-se o Microsoft Excel ${ }^{\circledR}$. O teste de KolmogorovSmirnov foi usado para testar a normalidade dos dados da plataforma dos grupos tratado e controle. Por meio do teste $t$, com nível de significância 5\%, fez-se a comparação da porcentagem de apoio de peso entre $\mathrm{D}$ e $\mathrm{E}$, entre $\mathrm{F}$ e $\mathrm{T}$, entre DD e DE, e entre TD e TE, dentro de grupos e entre grupos.

Para estabelecer o grau de associação entre os parâmetros utilizados para a avaliação da recuperação, foi realizada análise de correlação usando-se o teste Spearman Rank Order Correlation, com nível de significância 5\%, utilizando-se o programa estatístico Sigmastat ${ }^{\circledR}$.

\section{RESULTADOS E DISCUSSÃO}

O grupo-controle foi necessário para correlacionar os dados de apoio ou transferência de peso, pois os animais tratados não foram avaliados anteriormente ao procedimento cirúrgico. O exame radiográfico e a avaliação clínica desse grupo tiveram o objetivo de certificar a normalidade desses animais.

A modificação dos graus de claudicação descritos por Braden e Brinker (1973) foi realizada para estabelecer uma classificação mais condizente com a realidade dos animais avaliados, visto que, diferentemente desses autores, nos animais deste estudo observou-se que o apoio e o suporte do peso em estação foram uma das últimas etapas visualizadas no 
período de recuperação. Também foi necessária a separação do grau IV em dois graus, para melhor adequação dos pacientes nas classificações. Dessa forma, acrescentou-se mais um grau na avaliação, que passou de IV, descrita pelos autores citados, para V graus neste estudo.

Na classificação dos graus de claudicação, 16,7\% dos pacientes apresentaram grau III, 22,2\% grau IV e $61,1 \%$ grau V. Os pacientes do grau III, coincidentemente, foram os mesmos avaliados com menos de dois meses de pós-cirúrgico, levando a crer que ainda estavam em recuperação.

Para a avaliação dos animais na plataforma de força, primeiro foi necessário avaliar os animais do grupo-controle, para delimitar como foi o apoio normal em estação dos cães sobre a plataforma de força, pois os únicos dados disponíveis sobre uso de plataforma de força em cães foram obtidos em marcha. Como o enfoque deste trabalho foi visualizar a existência ou ausência de transferência de peso entre os membros, fez-se necessário o uso da plataforma em padrão ortostático, pois em estação os animais tendiam a retirar o peso de um membro que lhe causasse dor ou incômodo e transferi-lo para os demais. $\mathrm{O}$ ideal seria usar quatro plataformas de força para que se pudesse mensurar o peso dos quatro membros ao mesmo tempo. Porém, como é um equipamento de alto custo, verificou-se que é possível observar a transferência usando uma única plataforma.

Após se verificar a normalidade dos dados da porcentagem média de peso em cada um dos oito posicionamentos dos animais controle pelo teste de Kolmogorov-Smirnov (Programa Sigmastat), foram obtidos a média e o desvio-padrão por análise descritiva (Tab.2).

Tabela 2. Média, desvio-padrão, limite superior e limite inferior da porcentagem de peso no lado direito (D), lado esquerdo (E), dianteiras (F), traseiras (T), dianteira direita (DD), dianteira esquerda (DE), traseira direita (TD) e traseira esquerda (TE) obtidos na avaliação sobre a plataforma de força em padrão ortostático do grupo-controle

\begin{tabular}{|c|c|c|c|c|c|c|c|c|}
\hline & $\mathrm{D}$ & $\mathrm{E}$ & $\mathrm{F}$ & $\mathrm{T}$ & $\mathrm{DD}$ & $\mathrm{DE}$ & TD & TE \\
\hline Média & 47,11 & 52,89 & 63,565 & 36,435 & 33,145 & 31,77 & 18,747 & 16,337 \\
\hline Desvio-padrão & 4,559 & 4,559 & 3,244 & 3,244 & 4,835 & 3,899 & 5,455 & 2,691 \\
\hline Limite superior & 51,669 & 57,449 & 66,809 & 39,679 & 37,98 & 35,669 & 24,202 & 19,028 \\
\hline Limite inferior & 43,551 & 48,301 & 60,321 & 33,192 & 28,31 & 27,671 & 13,291 & 13,645 \\
\hline
\end{tabular}

Observou-se diferença significativa $(\mathrm{P}<0,05)$ entre o apoio dos membros torácicos e pélvicos, sendo em torno de $63,6 \%$ do peso apoiado nos membros torácicos e $36,4 \%$ nos pélvicos. Esse fato é condizente com a anatomia destes animais, pois se a cabeça e o tórax são as regiões mais pesadas, o maior apoio verifica-se sobre os membros torácicos.

Os dados de porcentagem de peso em cada um dos oito posicionamentos de cada animal do grupo tratado foram comparados aos do grupocontrole (Tab. 1), enquadrando os dados dentro dos limites superior e inferior estabelecidos. Os pacientes que apresentaram valores acima do limite superior em um determinado posicionamento foram considerados com aumento de peso sobre o membro ou membros em questão. Os pacientes com valores abaixo do limite inferior em um determinado posicionamento foram considerados com redução de peso sobre o membro ou membros em questão.

Em todos os animais avaliados em que ocorreu transferência, foi possível determinar o percentual de transferência, de onde foi retirado o peso e para onde foi transferido, somente utilizando a avaliação dos posicionamentos em separado de cada membro (DD, DE, TD e TE). As avaliações nos demais posicionamentos (D, $\mathrm{E}, \mathrm{F}$ e $\mathrm{T}$ ) foram utilizadas somente para conferência. Portanto, em estudos futuros, que utilizem a plataforma no padrão ortostático, recomenda-se o uso dos posicionamentos em separado de cada membro associado ao com as quatro patas sobre a plataforma, como metodologia, não sendo necessários os outros quatro posicionamentos, tornando mais rápida a avaliação sobre a plataforma de força.

Foi classificada como transferência direta aquela em que o animal reduzia o peso no membro 
afetado ou em ambos os membros pélvicos; e indireta, quando o animal reduzia o peso nos demais membros.

A amostra mostrou-se heterogênea em relação à porcentagem e ao destino da transferência de peso de cada animal. No intuito de homogeneizar a amostra, para posterior tratamento estatístico, foi estabelecido um padrão de transferência, em que os animais foram classificados conforme suas características semelhantes dentro dos respectivos grupos: ausência de transferência (TF0); transferência direta menor que $5 \%$ e indireta entre 5 e 10\% (TF1); transferência direta entre 5 e 10\% (TF2); transferência direta acima de $10 \%$ (TF3). Observou-se que 33,33\% dos animais foram classificados no grupo $0 ; 50 \%$ no grupo 1; 5,6\% no 2 e 11,1\% no grupo 3 . Estes últimos, cujo tempo decorrido da cirurgia era menor que dois meses, fortalecendo a sugestão de estarem em período de recuperação.

Os animais que apresentaram transferência de peso, ou seja, grupos 1 , 2 e 3, foram distribuídos segundo o membro afetado, para classificar qual o destino da transferência. Após a divisão, foi testada a normalidade dos dados que foi positiva, seguindo-se o teste $t(\mathrm{P}<0,05)$, para comparação de cada média de avaliação dos grupos: membro direito afetado (Dd, Ed, Fd, Td, DDd, DEd, TDd, Ted) e membro esquerdo afetado (De, Ee, Fe, Te, DDe, DEe, TDe, TEe) e grupo-controle (Dc, Ec, Fc, Tc, DDc, DEc, TDc, TEc).

Comprovou-se a transferência em ambos os grupos: no grupo com o membro direito afetado, o peso foi retirado do membro pélvico direito e distribuído nas dianteiras, e no grupo com o membro esquerdo afetado, o peso foi retirado do membro pélvico esquerdo e transferido para o lado contralateral.

Com relação à avaliação radiográfica da articulação coxofemoral, a literatura descreve que podem ser utilizados três posicionamentos diferentes na posição ventrodorsal. No primeiro os membros pélvicos são tracionados, com moderada abdução e ligeira rotação externa (Schebitz e Wilkens, 2000). No segundo os membros pélvicos sofrem extensão completa, com cada fêmur paralelo entre si e a coluna vertebral, e ligeira rotação interna - este é o posicionamento indicado nos casos de displasia, porém, para ser adequadamente realizado, é necessária anestesia geral ou sedação profunda do animal (Lust, 1998). O último posicionamento é menos doloroso para o paciente não sedado com patologia na região pélvica e, portanto, mais fácil de ser realizado que os descritos acima. Com as coxas colocadas em posição fisiológica, realizam-se flexão e abdução adequadas das articulações do joelho (Schebitz e Wilkens, 2000). No presente estudo, por se tratar de animais da rotina hospitalar, em que não seria apropriada sedação ou anestesia geral, optou-se pela associação de dois posicionamentos no exame radiográfico, para garantir a adequada visualização da articulação coxofemoral.

Foram utilizados o segundo e o terceiro posicionamentos descritos. Porém, a tração e rotação interna dos membros necessários eram realizadas até o ponto em que o animal permitia, sem manifestar desconforto, pois não estava sedado. Dessa forma, foi importante a realização do posicionamento com flexão e abdução para complementar o exame, uma vez que era menos doloroso e com melhor aceitação dos animais sem a sedação.

Poucos sistemas de escore radiográfico foram publicados para avaliação das articulações em cães (Roy et al., 1992). Portanto, neste estudo, o exame radiográfico nos grupos tratados teve por objetivo somar-se às demais avaliações para acrescentar informações, conforme Evers et al. (1997). Três animais (16,7\%) apresentaram alterações radiográficas condizentes com as demais avaliações. Estas complicações surgiram somente após longo tempo transcorrido da cirurgia, em média cinco anos e meio. Bone et al. (1984) observaram que os primeiros sinais da doença articular degenerativa não se tornaram aparentes até 16 meses após a cirurgia.

O primeiro animal apresentou imagem radiográfica compatível com necrose asséptica no mesmo membro em que, há mais de seis anos, fora realizada inserção de pino transacetabular. Este animal estava clinicamente sem alterações, mas com grau 1 de transferência, demonstrando que essa avaliação pode ser mais precisa. O segundo apresentou imagem compatível com displasia coxofemoral no membro contralateral, quando foi submetido à colocefalectomia há quase seis anos e grau IV de claudicação, 1 de transferência. O terceiro apresentou imagem compatível com uma esquírula óssea, na 
articulação coxofemoral onde foi realizada colocefalectomia há quase cinco anos e grau III de claudicação, 1 de transferência.

O grau de claudicação foi inversamente proporcional à transferência de peso, ou seja, à medida que se aumenta o grau de claudicação, diminui-se a transferência de peso. Este fato é condizente com o esperado, pois o aumento do grau de claudicação (I em direção a V) e a diminuição da transferência de peso (3 em direção a 0) estão diretamente relacionados à melhora do animal, assim como o inverso também é verdadeiro. A recuperação foi diretamente proporcional à transferência de peso e inversamente proporcional ao grau de claudicação, fato compreensível, já que o aumento do grau de claudicação (I em direção a V) e a diminuição da transferência de peso (3 em direção a 0) levaram à melhora da recuperação do animal.

O tempo foi indiretamente proporcional à transferência de peso e diretamente proporcional ao grau de claudicação e recuperação, fato condizente com o esperado, pois, conforme aumenta o tempo passado da cirurgia, a recuperação do animal é progressiva, tende a reduzir a transferência de peso (3 em direção a 0) e a aumentar o grau de claudicação (I em direção a V). Dessa forma, comprovou-se a eficácia do parâmetro transferência de peso após correlacioná-lo ao grau de claudicação e à recuperação dos animais.

A plataforma de força para avaliar a transferência de peso, apesar de ser um parâmetro novo para a veterinária, principalmente na metodologia em que foi usada, neste estudo mostrou-se eficiente, estabelecendo correlação com todos os demais parâmetros, além de demonstrar alterações que passaram despercebidas pelos outros parâmetros, como avaliação do grau de claudicação e exame radiográfico. Evans et al. (2005) afirmaram que a plataforma se mostrou um método acurado e mais sensível que a observação visual do grau de claudicação, pois, ao se comparar essas duas avaliações, $75 \%$ dos animais que se apresentavam clinicamente normais tiveram déficits demonstrados pela plataforma. Bockstahler et al. (2007) também mostraram a plataforma como um método, em longo prazo, mais sensível que o exame radiográfico na detecção de alterações displásicas na articulação coxofemoral subclínicas ainda não detectadas pela radiografia.

\section{CONCLUSÕES}

Por meio do grupo-controle, foi possível padronizar o uso da plataforma de força em padrão ortostático para cães, caracterizar a distribuição do peso corporal/apoio sobre os membros em animais normais e desenvolver uma metodologia adequada para esse tipo de coleta de dados sobre a plataforma de força na medicina veterinária. A plataforma de força em padrão ortostático, mediante determinação da distribuição do apoio e transferência de peso, mostrou ser um método objetivo e eficiente, algumas vezes mais sensível que os demais parâmetros de avaliação em pacientes ortopédicos com afecções articulares.

\section{REFERÊNCIAS BIBLIOGRÁFICAS}

ANDERSON, M. A.; MANN, F.A. Force plate analysis: a noninvasive tool for gait evaluation. Small Anim. Orthop., v.15, p.857-867, 1994.

BASTOS, A.G.D.; LIMA, M.A.M.T.; OLIVEIRA, L.F. Avaliação de pacientes com queixa de tontura e eletronistagmografia normal por meio de estabilometria. Rev. Bras. Otorrinolaringol., v.71, p.305-310, 2005.

BERTRAM, J.E. A.; LEE, D.V.; CASE, H.N. et al. Comparison of the trotting gaits of Labrador Retrievers and Greyhounds. Am. J. Vet. Res., v.61, p.832-838, 2000.

BOCKSTAHLER, B.A.; HENNINGER, W.; MULLER, M. et al. Influence of bordeline hip dysplasia on joint kinematics of clinically sound Belgian Shepherd dogs. Am. J. Vet. Res., v.68, p.271-276, 2007.

BONE, D.L.; WALKER, M.; CANTWELL, H. D.Traumatic coxo-femoral luxation in dogs: results of repair. Vet. Surg., v.13, p.263-270, 1984.

BRADEN, T.D.; BRINKER, W.O. Effect of certain internal fixation devices on functional limb usage in dogs. J. Am. Vet. Med. Assoc., v.162, p.642-646, 1973. 
CONZEMIUS, M.G.; APER, R.L.; CORTI, L.B. Short-term outcome after total elbow arthroplasty in dogs with severe, naturally occurring osteoarthritis. Vet. Surg., v.32, p.545552, 2003.

DOGAN, S.; MANLEY, P.A.; VANDERBY JR, R. et al. Canine intersegmental hip joint forces and moments before and after cemented total hip replacement. J. Biomech., v.24, p.397-407, 1991.

DUARTE, M.F. Análise estabilográfica da postura ereta humana quasi-estática. 2000. 86f. Tese (Livre Docência) - Escola de Educação Física e Esporte da Universidade de São Paulo, São Paulo, SP.

EVANS, R.; HORSTMAN, C.; CONZEMIUS, $M$. Accuracy and optimization of force platform gait analysis in Labradors with cranial cruciate disease evaluated at a walking gait. Vet. Surg., v.34, p.446-449, 2005.

EVERS, P.; JOHNSTON, G.R.; WALLACE, L.J. et al. Long terms results of treatment of traumatic coxo-femoral joint dislocation in dogs: 64 cases. J. Am. Vet. Med. Assoc., v.210, p.5964, 1997.

FERREIRA, E.A.G. Postura e controle postural: desenvolvimento e aplicação de método quantitativo de avaliação postural. 2005. 80f. Tese (Doutorado em Ciências) - Faculdade de Medicina da Universidade de São Paulo, São Paulo, SP.

JEVENS, D.J.; DE CAMP, C.E.; HAUPTMAN, $\mathrm{J}$. et al. Use of force-plate analysis of gait to compare two surgical techniques for treatment of cranial cruciate ligament rupture in dogs. Am. J. Vet. Res., v.57, p.389-393, 1996.

LOTH, E.A. Estudo da correlação entre posturografia dinâmica foam-laser e plataforma de força no teste de integração sensorial em adultos jovens normais. 2007. 70f. Dissertação (Mestrado em Distúrbios na Comunicação Humana) - Universidade Federal de Santa Maria, Santa Maria, RS.
LUST, G. Outras afecções ortopédicas: Displasia coxo-femoral em cães. In: SLATTER, D. Manual de cirurgia de pequenos animais. 2.ed. São Paulo: Manole, 1998. Cap.145, p.2286-2293.

OLIVEIRA, L.F. Estudo de revisão sobre a utilização da estabilometria como método de diagnóstico clínico. Rev. Bras. Eng., v.9, p.3753, 1993.

ROY, R.G.; WALLACE, L.J.; JOHNSTON, G.R. et al. A retrospective evaluation of stifle osteoarthritis in dogs with bilateral medial patella luxation and unilateral surgical repair. Vet Surg., v.21, p.475-479, 1992.

SCHEBITZ, H.; WILKENS, H. Atlas de anatomia radiográfica do cão e do gato. 5.ed. São Paulo: Manole, 2000, 244 p.

SUWANKONG, N.; MEIJ, B.P.; VAN KLAVEREN, N.J. et al. Assessment of descompressive surgery in dogs with degenerative lumbosacral stenosis using force plate analysis and questionnaires. Vet. Surg., v.36, p.423-431, 2007.

TEODORI, R.M.; GUIRRO, E.C.O.; SANTOS, R.M. Distribuição da pressão plantar e localização do centro de força após intervenção pelo método de reeducação postural global: Um estudo de caso. Fisiot. Mov., v.18, p.27-35, 2005.

TRÍPOLI, F.; MOREIRA, S. R.; OBERG, T. D. et al. Tarefas orientadas e biofeedback: efeitos na transferência de peso em hemiparéticos. Acta Fisiatr., v.15, p. 220-224, 2008.

VAN KLAVEREN, N.J.; SUWANKONG, N.; BOER, S. et al. Force plate analysis before and after dorsal descompression for treatmente of degenerative lumbosacral stenosis in dogs. Vet. Surg., v.34, p.450-456, 2005.

WHEELER, J.T.; RAISER, A.G. Artroplastia capsular de colonna modificada para cães - III Avaliação biomecânica. Cienc. Rural, v.22, p.7384, 1992. 